\title{
Preface: Autophagy and Oncogenesis
}

Autophagy is a process that cells use to control the degradation of accumulated unwanted and harmful cytoplasmic constituents. Ubiquitous in mammals and conserved throughout evolution, autophagy is a highly regulated system of membrane rearrangements that allows trafficking of cargoes to lysosomes, where their content is degraded by lysosomal hydrolysis and recycled. Autophagy's pleotropic functions include, among others, responding to cellular stress induced by nutritional deprivation, hypoxia, and metabolic misbalance by recycling depleted constituents. The three main categories of biologically similar lysosome-dependent processes are chaperone-mediated autophagy, microautophagy, and macroautophagy. The literature primarily focuses on the third category.

Autophagy is intrinsically involved in cellular development and therefore, has been the subject of numerous investigations concerning its role in cellular activation and the pathogenesis of several diseases, including cancer. In human cancers, depending on type and stage, autophagy has been shown to underlie both tumorigenesis and tumor suppression. Hence, the therapeutic application of agents that either inhibit or induce autophagy in cancer is the subject of intense research and is clinically challenging, although certain autophagy inhibitors in some cancers have been clinically tested .

This special issue of Critical Reviews ${ }^{\mathrm{TM}}$ in Oncogenesis, Autophagy and Oncogenesis, offers selective reviews of the role of autophagy in the pathogenesis of cancer. It contains seven reviews by highly experienced investigators on advancements in understanding of the conflicting roles of autophagy in cancer and potential applications of its inhibitors and inducers as therapeutic strategies.

Huang and associates (Chapter 1), in "Autophagy and Hallmarks of Cancer," introduce autophagy's role in tumor initiation and promotion, seeking to decipher its molecular regulatory mechanisms. They propose new therapeutics that target autophagy as a means of inhibiting malignant transformation and tumor progression. Moreover, they report on clinical trials using chloroquine and hydrochloroquine to inhibit autophagy in combination with other therapies for the treatment of resistant cancers. Notably, they highlight the need to develop more selective and specific autophagy inhibitors.

Sudharani (Chapter 2), in "Mechanism and Regulation of Autophagy in Cancer," reviews the contrasting roles of autophagy by exploring the molecular mechanisms that regulate this dichotomy and how to exploit them in the development of highly specific targeting agents for therapeutic purposes. These agents might be used in the clinic with other therapies in the treatment of highly resistant and metastatic cancers.

Huang and Bonavida (Chapter 3), in "A New Linkage between the Tumor Suppressor RKIP and Autophagy: Targeted Therapeutics," review the potential of signaling crosstalk between autophagy and the tumor-suppressing gene product Raf kinase inhibitor protein (RKIP). RKIP expression is significantly reduced or absent in the majority of cancers, and this has been associated with tumor growth, invasion, EMT, metastasis, and resistance to cytotoxic chemo-immunotherapies. Autophagy has also been implicated in the regulation of tumor cell response to chemo-immunotherapies. While many studies have investigated autophagy or cancer independently, few have looked into the relationship between RKIP and autophagy. In their review, Huang and Bonavida analyze RKIP and autophagy's coordinate regulatory and effector mechanisms. They propose pathways that suggest the involvement of several signaling modules - for example, p53, STAT-3, NF$\kappa \mathrm{B}$, and SNAIL - in the regulation of RKIP and the control of autophagy and vice versa.

Bonavida (Chapter 4), in "Linking Autophagy and the Dysregulated NF- $\kappa B$ /SNAIL/YY1/RKIP/ PTEN Loop in Cancer: Therapeutic Implications," reviews a possible link between autophagy regulation and the dysregulated NF-kB/SNAIL/YY1/RKIP circuit. Autophagy and the circuit are common phe- 
notypic features, controlled by both autophagy and the dysregulated circuit in the majority of cancers, that lead to cell proliferation, viability, invasion, EMT, metastasis, and resistance to cytotoxic therapies. Bonavida offers a link between autophagy and the circuit's various gene products and proposes that the targeting of any one gene product in the circuit and/or autophagy has the potential to be clinically beneficial in the treatment of primary and metastatic and resistant cancers.

Xiao and associates (Chapter 5), in "Driving Cytotoxic Natural Killer Cells into Melanoma: If CCL5 Plays the Music, Autophagy Calls the Shots," tackle the challenge of identifying a selected autophagyspecific protein as a target for cancer intervention. The majority of autophagy-related proteins perform no autophagic functions. The authors argue that several target proteins involved in early autophagy are potential candidates as its inhibitors, and they provide examples, such as the kinase Vps34 protein (a Class III PI3K involved in autophagy initiation). Small molecules that inhibit Vps34 have been developed and should be useful in combination with chemo- or immunotherapy. In particular, inhibition of autophagy may induce the infiltration of natural killer (NK) cells into the antitumor microenvironment and may mediate tumor immunity.

Marzvayan and associates (Chapter 6), in "Herbal Medicine in the Mitigation of ROS, Autophagy and Cancer: A Review," look at herbal medicines and supplements in autophagy regulation and cancer therapeutics. They review the most common herbs investigated and their impact on ROS (a mediator that regulates autophagy's contrasting roles in cancer), noting that all of them except ginseng promote ROS accumulation in cancer cells, thus inducing cancer cell apoptosis. They also note, however, that ROS levels dictate the outcome. They recommend preclinical in vitro studies to investigate both the therapeutic effects of herbal supplements on different cancer cell lines and the molecular mechanisms that underlie them.

Yang and associates (Chapter 7), in "Molecular Responses of Cancers by Natural Products: Modifications of Autophagy Revealed by Literature Analysis," extensively review, both analytically and quantitatively, studies on medicinal products in cancer research. They uncover an evolving trend in the increasing number of reports on natural products which focus most frequently on curcumin, flavonoids, and resveratrol.

This special issue on autophagy and oncogenesis delves into the most fundamental aspects of the relationship between autophagy and cancer on the one hand, and the translational application of autophagy inhibitors and inducers, on the other. The editor wishes to thank all contributors for their novel insights and imaginative proposals for future consideration. The editor also wishes to thank Ms. Jazelle Bautista for her great effort in editing and formatting the issue's content.

\section{Benjamin Bonavida, $\mathrm{PhD}$}

Department of Microbiology, Immunology and Molecular Genetics, David Geffen, School of Medicine, University of California, Los Angeles 